\title{
Physics-Based Large-Signal Sensitivity Analysis of Microwave Circuits Using Technological Parametric Sensitivity from Multidimensional Semiconductor Device Models
}

\author{
Fabrizio Bonani, Member, IEEE, Simona Donati Guerrieri, Fabio Filicori, \\ Giovanni Ghione, Senior Member, IEEE, and Marco Pirola
}

\begin{abstract}
The authors present an efficient approach to evaluate the large-signal (LS) parametric sensitivity of active semiconductor devices under quasi-periodic operation through accurate, multidimensional physics-based models. The proposed technique exploits efficient intermediate mathematical models to perform the link between physics-based analysis and circuit-oriented simulations, and only requires the evaluation of $\mathrm{dc}$ and ac small-signal (dc charge) sensitivities under general quasi-static conditions. To illustrate the technique, the authors discuss examples of sensitivity evaluation, statistical analysis, and doping profile optimization of an implanted MESFET to minimize intermodulation which make use of LS parametric sensitivities under two-tone excitation.
\end{abstract}

Index Terms-Microwave devices, nonlinear circuits, optimization methods, semiconductor device modeling, sensitivity, yield estimation.

\section{INTRODUCTION}

$\mathbf{T}$ HE PHYSICS-BASED design and optimization of monolithic microwave integrated circuits, (MMIC's), has been the object of growing interest during the last few years [1]. The physics-based design approach is particularly appealing, since it makes direct use of physical and geometrical input data, rather than of intermediate electrical parameters, as the variables to be tuned during circuit optimization. In particular, yield-driven optimization, which is of paramount importance in designing marketable MMIC's, is straightforward in the physics-based approach, since this avoids the complex modeling of the statistical correlations between the electrical performance variations induced by random changes in the (often uncorrelated) technological input parameters.

As discussed in [2], a fundamental block in an integrated technological computer-aided design (TCAD) environment leading from process data to electrical circuit simulation and design is the physics-based active device model. This model is needed to provide an accurate link between process

Manuscript received August 6, 1996; revised December 5, 1996. This work was supported in part by the Italian Ministry of Research through the MIA (Materiali Innovativi Avanzati) project and in part by the National Council of Research (CNR) through the project SAI (Sistemi autronici a iperfrequenze).

F. Bonani, S. Donati Guerrieri, G. Ghione, and M. Pirola are with the Dipartimento di Elettronica, Politecnico di Torino, 10129 Torino, Italy.

F. Filicori is with D.E.I.S., Università di Bologna, 40136 Bologna, Italy.

Publisher Item Identifier S 0018-9480(97)03100-1. data and electrical behavior. Unfortunately, accurate physical modeling of HF semiconductor devices cannot usually be achieved through computationally efficient, analytical, or quasi-analytical models. Instead, the numerical implementation of transport models in quasi-two-dimensional (2-D), full 2-D, or even three-dimensional (3-D) form is often needed to accurately foresee the device performances in dc, ac smallsignal, and large-signal (LS) periodic or quasi-periodic operation.

As is well known, an efficient TCAD environment should allow not only for performance evaluation, but also for device and circuit efficient parametric optimization and statistical analysis [3]. This requires that the physics-based models also estimate the effect of small variations of input technological and physical parameters on the electrical device performances, i.e., the device small-change sensitivity with respect to the process parameters under $\mathrm{dc}$ and small or LS ac operation. To this aim, linearization techniques must be applied to the model equations, as first proposed in 1987 by Baccarani et al., to evaluate the dc small-change device sensitivity with respect to uniform doping and geometry variations [4].

The analogy with the sensitivity evaluation in electrical networks [5] suggested the use of an adjoint approach in $\mathrm{dc}$ and ac small-signal device sensitivity computation, as proposed in [6] within the framework of a majority-carrier drift-diffusion GaAs FET model. To allow for the extension of the method to bipolar and nonstationary transport models, some of the present-day authors proposed a generalization of the adjoint approach, whose idea is derived from Branin's method for the sensitivity analysis of electrical networks [7]. The technique, first proposed with application to the multidimensional noise modeling of semiconductor devices [8], was then recently extended to the dc and ac small-signal sensitivity analysis and exploited for the optimization and statistical analysis of high-frequency (HF) devices [9] within the framework of a bipolar drift-diffusion model.

Little or no attention has been paid so far, to the authors' best knowledge, to the evaluation of the device sensitivities under LS, quasi-periodic excitation. Unfortunately, their direct computation through a physics-based multidimensional model requires, as a preliminary step, a LS analysis under periodic 
or quasi-periodic excitation. Although this can be addressed both through time-domain [10], [11] and frequency-domain techniques based on the harmonic-balance (HB) method [12], the computational intensity is great, and more complex cases (like incommensurate two-tone or multitone excitation) have not been addressed yet.

In [2], a different strategy was proposed to enable the efficient LS analysis under periodic excitation through physicsbased models. The basic idea was to exploit accurate circuitoriented intermediate models directly identified through the physics-based analysis of the (almost) intrinsic device. Owing to the availability of internal field and charge distributions and to the straightforward and exact deembedding of parasitics, circuit models can be extracted with excellent accuracy from physics-based simulations. Among these models, the nonlinear integral model (NIM) proposed in [13] is particularly well suited, since it only requires dc and small-signal data to be evaluated through the physical model; the accuracy of the NIM when compared to multidimensional simulation is excellent, as shown in [13]. Nevertheless, more conventional chargecontrol LS equivalent circuit models (which can be interpreted as limiting cases of the NIM) can also provide a very good link between physics-based and LS simulation, as discussed in [14].

In this paper, the authors make use of intermediate, circuitoriented, mathematical models to efficiently perform the LS sensitivity analysis under quasi-periodic excitation through multidimensional physics-based models. The computational burden is acceptable, since, as shown in Section III, the LS sensitivity analysis only requires the $\mathrm{dc}$ and ac small-signal sensitivity evaluation in several working points if the NIM is exploited as an intermediate model, and the dc sensitivity only if a quasi-static charge-control modeling approach is accurate enough in the operating frequency band. To demonstrate the method, the authors evaluate the LS sensitivities under two-tone excitation of a microwave FET by means of a 2-D drift-diffusion model coupled to a charge-control LS equivalent circuit, and develop (see Section IV) two case studies. The first one concerns the statistical analysis of the third-order intermodulation products (IMP's) with respect to random variations of the doping profile of an implanted device. The results obtained through the sensitivity approach are compared with the direct physics-based simulation and found in good agreement. In the second example, a twoimplant MESFET is optimized with respect to the intermodulation performance, thus demonstrating that the efficiency of the proposed technique makes the direct optimization of the device with respect to LS requirements computationally affordable.

\section{Small-Change Sensitivity Analysis}

The small-change sensitivity of an electrical device performance $\gamma$ with respect to a technological parameter $\sigma$ is defined as

$$
S_{\sigma}^{\gamma}=\frac{\partial \gamma}{\partial \sigma}
$$

In what follows, it shall be assumed that the device under consideration is a two-port and that $\gamma$ is an input (output) voltage, current, or charge. According to the nature of the parameter $\gamma$, the following types of sensitivities are introduced;

1) dc sensitivity, where $\gamma$ is a dc electrical parameter, like the output dc current or the dc charge stored under the gate in a FET;

2) ac small-signal sensitivity, where $\gamma$ is a small-signal parameter such as the elements of the frequency-dependent scattering or admittance matrix;

3) ac LS sensitivity, where $\gamma$ is the harmonic component of an input (output) device voltage or current under quasiperiodic operation, or related network functions (such as the ratio between the amplitude of the fundamentals and the third-order IMP's under two-tone excitation).

Since the devices considered (microwave FET's) are basically voltage-controlled, it shall be assumed that all sensitivities are defined at constant nominal applied voltage.

In the discussion, reference will be made to a physicsbased semiconductor device model. To fix the ideas, a 2-D drift-diffusion bipolar model will be considered, including Poisson, and the electron and hole continuity equations. As mentioned in [2], the drift-diffusion model, despite its wellknown limitations in the treatment of submicron devices, can still be assumed as the basis for a quantitative discussion of the device behavior versus the technological parameters. In fact, nonstationary transport effects can be (at least approximately) allowed for through heuristical models [2]. Moreover, all the techniques described in this paper can, in principle, be extended to nonstationary transport models. The drift-diffusion model equations are well known (see e.g., [2] and references therein) and will not be repeated here; one need only recall that the model unknowns are the electron and hole densities $n(\underline{r}, t), p(\underline{r}, t)$, and the potential distribution $\psi(\underline{r}, t)$.

Let one assume that a suitable spatial discretization scheme [15] has been applied to the model partial differential equations, so as to obtain a system of $3 \times N$ time-domain ordinary differential equations, where $N$ is the number of discretization nodes. The discretized unknowns are arrays of time-dependent nodal values for electron and hole concentrations and electric potential, which shall be indicated, for the sake of brevity, as $n, p, \psi$ (i.e., with the same symbol as the corresponding variable). To enable a formal treatment, the discretized equations and boundary conditions are denoted as

$$
\left\{\begin{array}{l}
\underline{F}(\psi, n, p, \dot{n}, \dot{p} ; \sigma)=0 \\
\underline{\chi}(\psi, n, p ; s, \sigma)=0
\end{array}\right.
$$

where $\underline{F}=\left\{F^{(\psi)}, F^{(n)}, F^{(p)}\right\}=0$ includes the discretized Poisson, electron continuity, and hole continuity equations, respectively. The equation $\underline{\chi}=\left\{\chi^{(\psi)}, \chi^{(n)}, \chi^{(p)}\right\}=0$ denotes the discretized boundary condition set (of dimension $3 \times M$, where $M$ is the number of device terminals); $\dot{\alpha}=$ $\partial \alpha / \partial t(\alpha=n, p)$ and $s(t)$ is a set of external driving current or voltage sources. The device equations and boundary conditions depend on the parameter set $\sigma$, relative to physical and technological data such as mobility models, doping, and device dimensions. 


\section{A. Evaluation of dc and Small-Signal ac Sensitivities}

The evaluation of the dc and small-signal ac sensitivities through a physics-based multidimensional model is discussed in detail in [9]; here, only a few conclusions will be provided, relevant to the ensuing treatment of LS sensitivities. As shown in [9], the dc and small-signal ac sensitivities can be derived by linear perturbation of the device model around a nominal dc working point. Assume that in (1), the electrical source term includes a dc bias $s_{0}$ superimposed to small-amplitude timevarying ac generators $\hat{s}(t)$, i.e., $s=s_{0}+\hat{s}(t)$; suppose now that the parameter set $\sigma$ undergoes a small time-independent variation $\Delta \sigma$ with respect to its nominal value $\sigma_{0}$, so that $\sigma=$ $\sigma_{0}+\Delta \sigma$. The resulting potential and carrier concentrations can be written as

$$
\alpha(t)=\alpha_{0}+\Delta \alpha+\hat{\alpha}(t)+\Delta \hat{\alpha}(t), \quad \alpha=\psi, n, p
$$

where $\alpha_{0}$ is the dc response with nominal parameters, $\Delta \alpha$ the dc perturbation due to the parameter variation $\Delta \sigma, \hat{\alpha}(t)$ the small-signal response to $\hat{s}(t)$ with nominal parameters and $\Delta \hat{\alpha}(t)$ is the small-signal perturbation due to the parameter variation. Following the discussion in [6], a linear perturbation approach can be exploited whereby $\Delta \alpha$ is proportional to $\Delta \sigma$, $\hat{\alpha}(t)$ to $\hat{s}(t)$, while $\Delta \hat{\alpha}(t)$ is a bilinear function of $\hat{s}(t)$ and $\Delta \sigma$. The dc perturbation can be obtained from a linear system derived by a Taylor expansion of (1) around the nominal dc solution

$$
\begin{aligned}
& \sum_{\alpha=\psi, n, p} F_{\alpha}^{(\beta)} \Delta \alpha=-F_{\sigma}^{(\beta)} \Delta \sigma \\
& \sum_{\alpha=\psi, n, p} \chi_{\alpha}^{(\beta)} \Delta \alpha=-\chi_{\sigma}^{(\beta)} \Delta \sigma
\end{aligned}
$$

where $F_{\alpha}^{(\beta)}=\partial F^{(\beta)} / \partial \alpha$ and $\chi_{\alpha}^{(\beta)}=\partial \chi^{(\beta)} / \partial \alpha(\alpha, \beta=$ $\psi, n, p)$ are evaluated at the dc working point. By expressing the small-signal model in the frequency domain, where $\tilde{\alpha}(\omega)$ is the Fourier transform of $\hat{\alpha}(t)(\alpha=\psi, n, p, s), \tilde{F}_{\alpha}^{(\beta)}=$ $\tilde{F}_{\alpha}^{(\beta)}(\omega)=F_{\alpha}^{(\beta)}+j \omega F_{\dot{\alpha}}^{(\beta)}(\alpha=n, p)$ and $\tilde{F}_{\psi}^{(\beta)}=F_{\psi}^{(\beta)}$, one obtains that the parametric variation of the ac small-signal response is

$$
\begin{aligned}
\sum_{\alpha=\psi, n, p} \tilde{F}_{\alpha}^{(\beta)} \Delta \tilde{\alpha}(\omega) & =S_{F}^{(\beta)} \\
\sum_{\alpha=\psi, n, p} \chi_{\alpha}^{(\beta)} \Delta \tilde{\alpha}(\omega) & =S_{\chi}^{(\beta)}
\end{aligned}
$$

where $\beta=\psi, n, p$ and the source terms are bilinear functions of the small-signal solution and of the dc parametric variations

$$
\begin{aligned}
& S_{F}^{(\beta)}=\sum_{\zeta=\psi, n, p} \sum_{\xi=\psi, n, p, \sigma} \tilde{\zeta}(\omega) \tilde{F}_{\xi \zeta}^{(\beta)} S_{\sigma}^{\xi} \Delta \sigma \\
& S_{\chi}^{(\beta)}=\sum_{\zeta=\psi, n, p, s} \sum_{\xi=\psi, n, p, \sigma} \tilde{\zeta}(\omega) \chi_{\xi \zeta}^{(\beta)} S_{\sigma}^{\xi} \Delta \sigma
\end{aligned}
$$

and where $\beta=\psi, n, p, \quad \tilde{F}_{\xi \zeta}^{(\beta)}=\tilde{F}_{\xi \zeta}^{(\beta)}(\omega)=F_{\xi \zeta}^{(\beta)}+$ $j \omega F_{\xi \dot{\zeta}}^{(\beta)}(\xi=\psi, n, p, \sigma, \zeta=n, p), \widetilde{F}_{\xi \psi}^{(\beta)}=F_{\xi \psi}^{(\beta)}(\xi=$ $\psi, n, p, \sigma), S_{\sigma}^{\sigma}=1$ and $\chi_{\sigma s}^{(\beta)}=0$. As $\tilde{\alpha}(\alpha=\psi, n, p)$ is proportional to $\tilde{s}(\omega)$, the source terms (5) ultimately are bilinear functions of $\tilde{s}(\omega)$ and $\Delta \sigma$, i.e., are proportional to $\tilde{s}(\omega) \Delta \sigma$. It should be noticed, however, that these source terms also depend on the dc sensitivities of the potential, electron, and hole distributions on the device volume.

In the direct approach to sensitivity evaluation, systems (3) and (4) are solved, and from the perturbations in the potential and carrier distributions the resulting perturbations in the short-circuit currents or open-circuit voltages at the device terminals are computed. This process has to be repeated $N_{p}$ times; with $N_{p}$ being the number of parameters involved. A more efficient approach is discussed in [6], [9], where the explicit solution of the perturbed systems is avoided and only one small-signal analysis on the adjoint system [6] or on a properly transposed system [9] are needed. Basically, both techniques exploit the fact that only the perturbation response at the device terminals (not throughout the device volume) is needed for a full small-change sensitivity characterization; the idea is similar altogether to the network sensitivity analysis method in [5]. Nevertheless, while the variation of the $\mathrm{dc}$ response is caused by a perturbation source term depending on the dc analysis with nominal parameters alone, for the ac sensitivity analysis one must evaluate the response to an ac source term which depends on the sensitivities of the internal potential and mobile charge distributions. Such sensitivities cannot be evaluated as in [6], [9], but require the direct solution of the system (3) for each of the $N_{p}$ parameters involved. It can, therefore, be concluded that while the dc sensitivity analysis can be carried out (through the methods in [6], [9]) and with negligible overhead with respect to the dc analysis alone, the ac small-signal sensitivity analysis intrinsically requires a fairly greater computational effort.

\section{B. Large-Signal Sensitivity Analysis Under Quasi-Periodic Excitation}

In order to introduce the small-change LS sensitivity analysis, one notices that under LS quasi-periodic excitation, the physical model can be written as

$$
\left\{\begin{array}{l}
\underline{F}\left[\psi(t), n(t), p(t), \dot{n}(t), \dot{p}(t) ; \sigma_{0}\right]=0 \\
\underline{\chi}\left[\psi(t), n(t), p(t) ; s(t), \sigma_{0}\right]=0
\end{array}\right.
$$

where $s(t)$ is a quasi-periodic excitation including a dc component and one or more tones. Suppose that the parameter undergoes a time-invariant perturbation $\Delta \sigma$; then, the system (6) can be linearized around the instantaneous working point, yielding a linear time-varying system with quasi-periodic coefficients in the time-varying perturbation $\Delta \alpha(t), \alpha=$ $\psi, n, p$ :

$$
\left\{\begin{array}{l}
\underline{F}_{\phi}(t) \Delta \psi(t)+\underline{F}_{n}(t) \Delta n(t)+\underline{F}_{p}(t) \Delta p(t) \\
\quad+\underline{F}_{\dot{n}}(t) \Delta \dot{n}(t)+\underline{F}_{\dot{p}}(t) \Delta \dot{p}(t)=-\underline{F}_{\sigma}(t) \Delta \sigma \\
\underline{\chi}_{\phi}(t) \Delta \psi(t)+\underline{\chi}_{n}(t) \Delta n(t)+\underline{\chi}_{p}(t) \Delta p(t) \\
\quad+\underline{\chi}_{\dot{n}}(t) \Delta \dot{n}(t)+\underline{\chi}_{\dot{p}}(t) \Delta \dot{p}(t)=-\underline{\chi}_{\sigma}(t) \Delta \sigma .
\end{array}\right.
$$


Thus, the frequency-domain solution is straightforward in terms of the harmonic components of the perturbation. If the LS system (6) has been solved through HB algorithms, the sensitivity system matrix in (7) simply is, in the frequency domain, the Jacobian required to carry out the LS solution according to a Newton scheme. Since the harmonic components of the perturbation are linearly related to the parameter variation, the small-change LS sensitivities are immediately recovered from the solution of system (7).

\section{LS SENSITIVITY UNDER PERIODIC EXCITATION THROUGH INTERMEDIATE CIRCUIT-ORIENTED MODELS}

The procedure outlined in the previous section to evaluate LS sensitivities includes a computationally intensive step, i.e., the solution of (6), requiring either a time-consuming time-domain analysis, or a frequency-domain HB approach [12]. To overcome this difficulty, a different approach, based on circuit-oriented behavioral models as intermediate links between physics-based device simulations and circuit analysis algorithms, can be conveniently adopted.

In particular, according to the approach in [2], [14], the LS circuit analysis is carried out through circuit-oriented nonlinear models and their characteristic parameters are extracted from dc and small-signal ac physics-based device simulations. In this way, LS circuit analysis is performed with good computational efficiency, while the link between physical parameters and the corresponding circuit performance is still provided by an off-line, physics-based device sensitivity analysis. In such conditions, LS circuit sensitivity analysis versus physical parameters implies a straightforward "chain derivative" sequence involving:

- derivatives of dc and small-signal ac characteristics versus physical parameters, i.e., physics-based device sensitivity analysis;

- derivatives of model parameters versus variations in dc and small-signal ac characteristics;

- derivatives of circuit electrical performances versus model parameters, i.e., conventional HB LS circuit sensitivity analysis versus model parameters [3].

In such a context, both conventional nonlinear equivalent circuits and mathematical "black-box" models (e.g., measurement-based look-up table models, like the nonlinear integral model [13] or the Root model [16]) could be used. However, the equivalent circuit approach is not well suited for parametric sensitivity analysis, since parameter extraction is normally based on numerical optimization procedures, which provide a nonexplicit, complex link between physics-based device simulation and equivalent circuit parameters. For this reason, the second step of the chain derivative sequence would be quite difficult to carry out. Instead, a look-up-table mathematical model like the NIM [13] is particularly attractive in this perspective, since it provides a direct, closed-form link between the LS behavior and the $\mathrm{dc}$ and bias-dependent small-signal ac characteristics of the device. According to this model, the LS current response can be predicted by using the following matrix equation:

$$
\begin{aligned}
& i(t)=I_{\mathrm{dc}}[v(t)]+\sum_{k=-M}^{M} \tilde{Y}\left[v(t), \omega_{k}\right] V_{k} e^{j \omega_{k} t} \\
& v(t)=\sum_{k=-M}^{M} V_{k} e^{j \omega_{k} t}
\end{aligned}
$$

where $i(t), v(t)$ are the currents and voltages at the device ports and $I_{\mathrm{dc}}$ are the nonlinear algebraic functions describing the dc characteristics of the device. The frequency-domain summation provides the strictly dynamic components of the currents in terms of the nonlinearly voltage-controlled dynamic admittance matrix $\tilde{Y}[v(t), \omega]=Y[v(t), \omega]-Y[v(t), 0]$, where $Y[v(t), \omega]$ is the bias-voltage-dependent small-signal admittance matrix [13].

As discussed in detail in [13], the model (8) is accurate if the "short-term memory" condition is satisfied; this assumption is related to (but less strong than) the well-known quasi-static approximation often exploited in device modeling. The shortterm memory requirement is commonly verified for "intrinsic" devices, i.e., devices not affected by dominating parasitic effects. Such a case occurs in physics-based models, wherein parasitics can be exactly deembedded [14]. Actually, a number of validation tests have shown good agreement between 2-D simulated LS responses and the corresponding values predicted according to (8).

On the basis of the mathematical model (8), the authors now address the issue of LS sensitivity evaluation. Direct differentiation of (8) leads to the following incremental equation:

$$
\begin{aligned}
\Delta i(t)= & \frac{\partial I_{\mathrm{dc}}}{\partial \sigma} \Delta \sigma+\frac{\partial I_{\mathrm{dc}}}{\partial v} \Delta v(t) \\
& +\sum_{k=-M}^{M} \tilde{Y}\left[v(t), \omega_{k}\right] \Delta V_{k} e^{j \omega_{k} t} \\
& +\sum_{k=-M}^{M}\left\{\frac{\partial \tilde{Y}}{\partial v} \Delta v(t)+\frac{\partial \tilde{Y}}{\partial \sigma} \Delta \sigma\right\} V_{k} e^{j \omega_{k} t}
\end{aligned}
$$

where all the derivatives are evaluated at the nominal operating conditions and where the device dc and small-signal ac sensitivities $\left(\partial I_{\mathrm{dc}} / \partial \sigma\right.$ and $\partial \tilde{Y} / \partial \sigma$, respectively) can be computed according to the previously outlined techniques. The derivatives $\partial I_{\mathrm{dc}} / \partial v$ simply are the bias-dependent elements of the admittance matrix in dc. The derivatives $\partial \tilde{Y} / \partial v$ of the dynamic admittance matrix elements with respect to the working point can be evaluated through a look-up-table approach.

This equation, together with HB-oriented algorithms for circuit sensitivity analysis (see e.g., [3] and references therein), allows LS sensitivity to be computed with far greater computational efficiency than through direct use of physics-based models. However, the use of (9) also requires (for the computation of $\partial \tilde{Y} / \partial \sigma$ ), the evaluation of ac sensitivities, whose high computational intensity has already been stressed.

In order to further reduce the computational effort, a more conventional approach can be adopted. In fact, if the simplifying assumption is made that the charge distribution depends in 
a quasi-static way on the applied voltages, the nonlinear dynamic admittances in (8) can be replaced by purely capacitive terms

$$
\tilde{Y}[v(t), \omega]=j \omega C[v(t)] .
$$

Thus, (8) is reduced to

$$
\begin{aligned}
i(t) & =I_{\mathrm{dc}}[v(t)]+\sum_{k=-M}^{M} C[v(t)] j \omega_{k} V_{k} e^{j \omega_{k} t} \\
& =I_{\mathrm{dc}}[v(t)]+\frac{d}{d t}\{Q[v(t)]\}
\end{aligned}
$$

with $C(v)=\partial Q / \partial v$. This is equivalent to approximating the purely dynamic component of the currents [represented by the summation in (8)], by a set of displacement currents, corresponding to a lumped, parallel $R C$ nonlinear equivalent circuit, which is a special case of the NIM equation (8). In such conditions, the corresponding incremental sensitivity model is obtained:

$$
\begin{aligned}
\Delta i(t)= & \frac{\partial I_{\mathrm{dc}}}{\partial \sigma} \Delta \sigma+\frac{\partial I_{\mathrm{dc}}}{\partial v} \Delta v(t) \\
& +\frac{d}{d t}\left\{\frac{\partial Q}{v} \Delta v(t)+\frac{\partial Q}{\partial \sigma} \Delta \sigma\right\}
\end{aligned}
$$

which clearly involves only first-order sensitivity calculations of the dc currents and of the internal charge distributions. In (12), the perturbation current $\Delta i(t)$ can be expressed as

$$
\Delta i(t)=\Delta i_{0}(t)+\frac{\partial I_{\mathrm{dc}}}{\partial v} \Delta v(t)+\frac{d}{d t}\left\{\frac{\partial Q}{\partial v} \Delta v(t)\right\}
$$

where

$$
\Delta i_{0}(t)=\frac{\partial I_{\mathrm{dc}}}{\partial \sigma} \Delta \sigma+\frac{d}{d t} \frac{\partial Q}{\partial \sigma} \Delta \sigma
$$

is a time-varying impressed current generator linearly dependent on the parameter variation $\Delta \sigma$.

In the present case, the authors address the simulation of microwave FET's. It is assumed that the nonlinear model is exploited to describe the intrinsic or quasi-intrinsic device, while parasitic elements (derived from the simulation or from measurements) are included in the embedding parasitic network shown in Fig. 1, where, for simplicity, the device is connected to an input voltage generator with internal impedance $Z_{G}(\omega)$ and to an output load with impedance $Z_{L}(\omega)$. The circuit also includes two bias input and output voltage generators $E_{\mathrm{IN}}$ and $E_{\text {OUT. }}$ The LS gate and drain (input and output) currents for the intrinsic device are expressed as

$$
\begin{aligned}
i_{G}(t) & =\frac{\partial Q}{\partial t} \\
i_{D}(t) & =I_{D}+\frac{\partial Q}{\partial V_{\mathrm{DS}}} \frac{d\left(v_{\mathrm{GS}}-v_{\mathrm{DS}}\right)}{d t}
\end{aligned}
$$

where $Q\left(V_{\mathrm{GS}}, V_{\mathrm{DS}}\right)$ is the "static" gate charge, and $I_{D}\left(V_{\mathrm{GS}}, V_{\mathrm{DS}}\right)$ is the de current. Thus, the LS sensitivity to any parameter $\sigma$ can be computed by using, in the framework

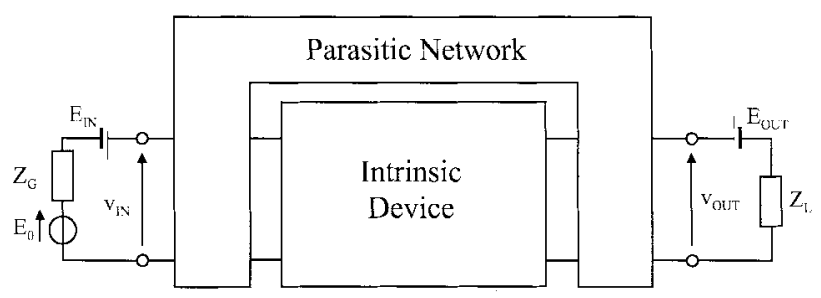

Fig. 1. Equivalent circuit used in the LS analysis of the active two-port.

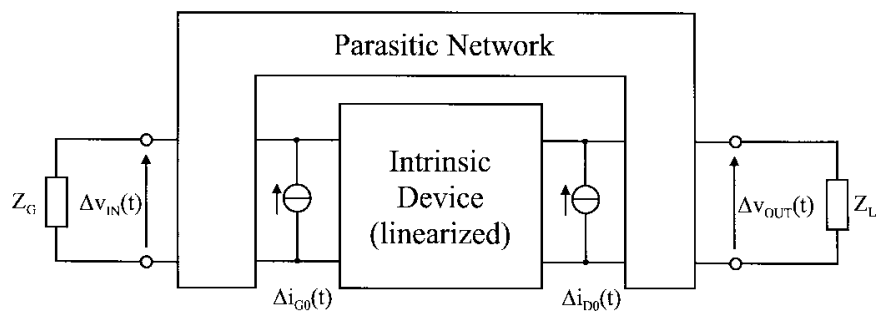

Fig. 2. Time-varying linear circuit used in the LS sensitivity analysis of the active two-port.

of HB-based techniques for circuit sensitivity analysis [3], the associated sensitivity model

$$
\begin{aligned}
\Delta i_{G}(t)= & \Delta i_{G 0}(t) \\
& +\frac{d}{d t}\left\{\frac{\partial Q}{\partial V_{\mathrm{GS}}} \Delta v_{\mathrm{GS}}(t)+\frac{\partial Q}{\partial V_{\mathrm{DS}}} \Delta v_{\mathrm{DS}}(t)\right\} \\
\Delta i_{D}(t)= & \Delta i_{D 0}(t)+\frac{\partial I_{D}}{\partial V_{\mathrm{GS}}} \Delta v_{\mathrm{GS}}(t)+\frac{\partial I_{D}}{\partial V_{\mathrm{DS}}} \Delta v_{\mathrm{DS}}(t) \\
& +\frac{\partial Q}{\partial V_{\mathrm{DS}}} \frac{d\left(\Delta v_{\mathrm{GS}}-\Delta v_{\mathrm{DS}}\right)}{d t}
\end{aligned}
$$

where the impressed time-varying perturbation current sources are:

$$
\begin{aligned}
\Delta i_{G 0}(t) & =\frac{d}{d t} \frac{\partial Q}{\partial \sigma} \Delta \sigma=\frac{d S_{\sigma}^{Q}}{d t} \Delta \sigma \\
\Delta i_{D 0}(t) & =\frac{\partial I_{D}}{\partial \sigma} \Delta \sigma+\frac{\partial^{2} Q}{\partial \sigma \partial V_{\mathrm{DS}}} \frac{d\left(v_{\mathrm{GS}}-v_{\mathrm{DS}}\right)}{d t} \Delta \sigma \\
& =S_{\sigma}^{I_{D}} \Delta \sigma+\frac{\partial S_{\sigma}^{Q}}{\partial V_{\mathrm{DS}}} \frac{d\left(v_{\mathrm{GS}}-v_{\mathrm{DS}}\right)}{d t} \Delta \sigma .
\end{aligned}
$$

It should be noted that the source terms only involve firstorder sensitivity calculations of quasi-static parameters, while the sensitivity analysis simply corresponds to the solution of the perturbation linear time-varying circuit in Fig. 2. As is well known from HB circuit analysis, the solution of the sensitivity network is straightforward once the Jacobian matrix relative to the HB process has been evaluated.

In conclusion, the present approach for LS sensitivity analysis allows for a dramatic improvement in terms of computational efficiency, while preserving a direct, closed-form link between circuit simulation and physics-based modeling.

As shown in the following section, the present technique for the first time (as far as the authors' knowledge goes) enables the direct, gradient-based LS performance optimization in the space of the active device technological parameters. 


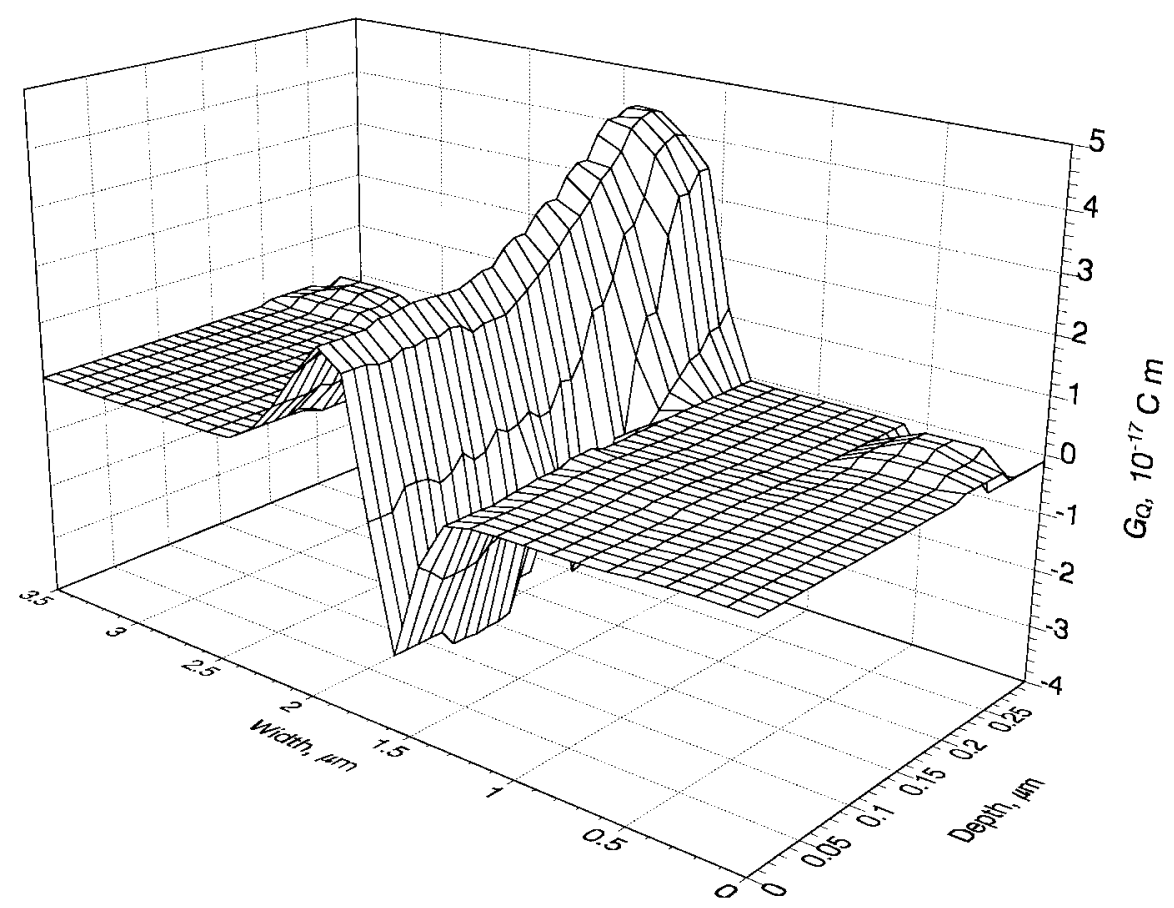

Fig. 3. Small-change variation of the gate charge (normalized versus the gate width) due to a local unit doping variation in the device cross section (see text) for a $0.5-\mu \mathrm{m}$ gate length MESFET. The bias point is $V_{\mathrm{DS}}=3 \mathrm{~V}$ and $V_{\mathrm{GS}}=-0.4 \mathrm{~V}$. The device terminals (upper surface) are located at $0.3-\mu \mathrm{m}$ depth; the source is between $0-0.5 \mu \mathrm{m}$, the gate between $1.5-2 \mu \mathrm{m}$, and the drain between $3-3.5 \mu \mathrm{m}$.

\section{EXAMPLES}

In this section, the authors present examples of application of the techniques discussed in this paper. As the basic design parameter for microwave MESFET's the authors chose the doping profile, which is the only technological feature that the device or circuit designer can change with a comparatively high degree of freedom. Geometrical parameters like the gate length are not realistic candidates for device optimization, although the related sensitivity can be exploited to model the effect of random process variations on the electrical device performances. The examples will be presented as follows. In Section IV-A, the authors describe some results concerning the LS device sensitivity per se, with particular attention on the concept of distributed sensitivity on the device volume, and on the frequency behavior of the LS sensitivities of input and output voltage harmonic components under two-tone excitation. In Section IV-B, the LS sensitivity is exploited to perform a statistical analysis of the amplitude variation of the third-order IMP's due to a stochastic spreading in the implantation energy of a single-implant device. Finally, Section IV-C, demonstrates the feasibility of the gradientbased optimization of the doping profile with the goal of minimizing the amplitude of the third-order IMP's due to a two-tone excitation of a GaAs MESFET.

In all examples discussed, the device sensitivities are computed by means of a 2-D drift-diffusion two-carrier III-V device simulator developed at Politecnico di Torino [9]. The HB analysis of the device under LS operation has been performed offhand on the basis of the equivalent circuit shown in Fig. 1, wherein the intrinsic device is described through a look-up table generated by the physics-based simulator. Look- up tables for the charge stored under the gate, the dc current, and the related sensitivities were computed in a proper set of bias points, then approximated through least-squares cubic splines. The spline interpolation of the look-up tables was also used to consistently compute the partial derivatives required to express the currents [see (15) and (16)]. The look-up table model was included in a program for LS circuit analysis under incommensurate multitone excitation implementing the technique of random sampling [17]; the same approach was used in evaluating the LS circuit sensitivity. In all computations shown, all frequencies were included up to the fifth harmonic of the highest input tone.

\section{A. Distributed Device Sensitivity and Frequency Behavior}

As a first example, the authors simulated a $0.5-\mu \mathrm{m}$ epitaxial GaAs MESFET, with 300- $\mu \mathrm{m}$ gate width and a (constant) doping level $N_{D}=10^{17} \mathrm{~cm}^{-3}$ in the epitaxial layer of thickness $0.2 \mu \mathrm{m}$. The $0.1-\mu \mathrm{m}$ substrate was considered semiinsulating; the gate-drain and gate-source spacing was $1 \mu \mathrm{m}$.

Fig. 3 shows an intermediate, but physically significant, result from the evaluation of the dc sensitivity of gate charge (see [9] for a similar example concerning the dc current). The working point is $V_{\mathrm{DS}}=3 \mathrm{~V}, V_{\mathrm{GS}}=-0.4 \mathrm{~V}$. To fix the ideas, let us call $G_{Q}(\underline{r})$ the function presented in Fig. 3, where $\underline{r}$ is a point on the device cross section; $G_{Q}\left(\underline{r}_{i}\right)$ is the variation in the charge due to a local unit variation of doping occurring in $\underline{r}_{i}$. By multiplying $G_{Q}$ by a space-dependent doping variation $\Delta N_{D}=\left(\partial N_{D} / \partial \sigma\right) \Delta \sigma$, where $\sigma$ is a doping parameter (e.g., the implant energy), the function

$$
s_{\sigma}^{Q}=G_{Q}(\underline{r}) \frac{\partial N_{D}}{\partial \sigma}
$$


gives the so-called distributed sensitivity [6], [9], whose integral on the device cross section finally yields the charge sensitivity. ${ }^{1}$ The effect of a localized doping variation is significant only in the region under the gate, while, as expected, little or no effect is detected for variations in the ohmic regions outside the intrinsic device.

The same device was used to evaluate the effect of a $1 \%$ doping variation on the harmonic components of the input and output voltages. The device was included in the circuit shown in Fig. 1 with $Z_{L}=Z_{G}=50 \Omega$ and the LS analysis was performed with a two-tone excitation of peak amplitude equal to $0.3 \mathrm{~V}$ around a dc working point $V_{\mathrm{GS}}=-1.4$ $\mathrm{V}, V_{\mathrm{DS}}=1.5 \mathrm{~V}$. Then, the intrinsic device was linearized around the quasi-periodic working point (see the circuit of Fig. 2 ). The analysis was performed by varying the fundamental frequency $f_{1}$ of the lower tone, while the distance between the two tones was kept constant so that $f_{2}=f_{1}+100 \mathrm{MHz}$. Fig. 4 shows the amplitude of the variation induced on the fundamental $f_{1}$, second harmonic $2 f_{1}$, and third-order IMP $2 f_{1}-f_{2}$ component of, respectively, $V_{\mathrm{IN}}$ and $V_{\text {OUT }}$. The resulting behavior is, for $V_{\mathrm{IN}}$, consistent with the capacitive nature of the device input. The output behavior is slightly more involved. A simplified model suggests that the decreasing sensitivity at high frequency is correlated to the decrease in voltage gain.

\section{B. Sensitivity-Based Statistical Analysis}

The linear approximation was applied to the LS statistical analysis of a single-implant GaAs MESFET whose geometry is the same as of the device described in Section IV-A. The implanted profile is Gaussian, with a dose $D=2 \times 10^{12}$ $\mathrm{cm}^{-2}$ and an implant energy $E=90 \mathrm{keV}$. This value was chosen since it corresponds to a minimum in the amplitude of the third-order IMP's of the output voltages for two input tones having $0.5 \mathrm{~V}$ peak amplitude around the working point $V_{\mathrm{GS}}=-1.4 \mathrm{~V}, V_{\mathrm{DS}}=1.5 \mathrm{~V}$, at frequencies $f_{1}=10$ $\mathrm{GHz}$ and $f_{2}=f_{1}+100 \mathrm{MHz}$ and with the loading circuit $Z_{L}=Z_{G}=50 \Omega$.

The authors modeled the implant energy as a random variable with Gaussian distribution around the mean value of $90 \mathrm{keV}$, and standard deviation of $2.5 \mathrm{keV}$. A Monte Carlo analysis was performed on the physics-based model, and then compared with the analytical distribution obtained, in the linear approximation, from the sensitivity evaluated at the mean energy. The implant energy dependence of the projected range and straggle was modeled as in [18]. The results from the Monte Carlo and sensitivity analyses are shown in Fig. 5. The agreement between the two approaches is satisfactory; the slight asymmetry in the exact distributions is consistent with the behavior of the sine and cosine amplitudes and of the total magnitude with respect to the implant energy, shown in Fig. 6.

\section{Gradient-Based Parametric Optimization}

As a final example, the authors performed the doping profile optimization of a two-implant MESFET with the aim of

\footnotetext{
${ }^{1}$ The function $G_{Q}$ is the Green's function (normalized by the gate width) relative to a charge point source, see [6], [9].
}

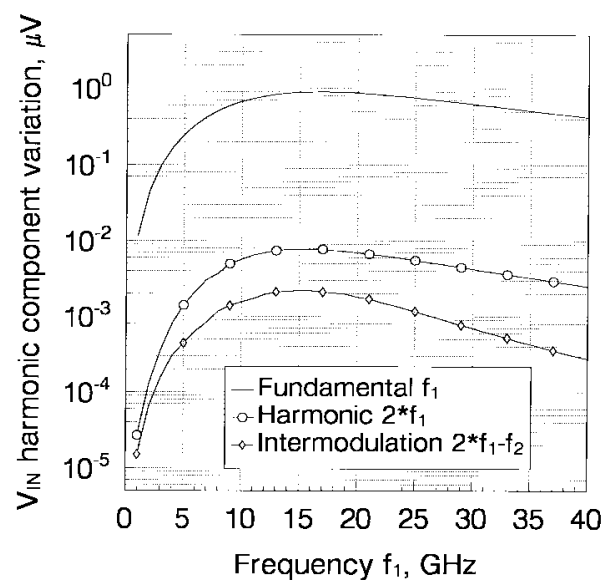

(a)

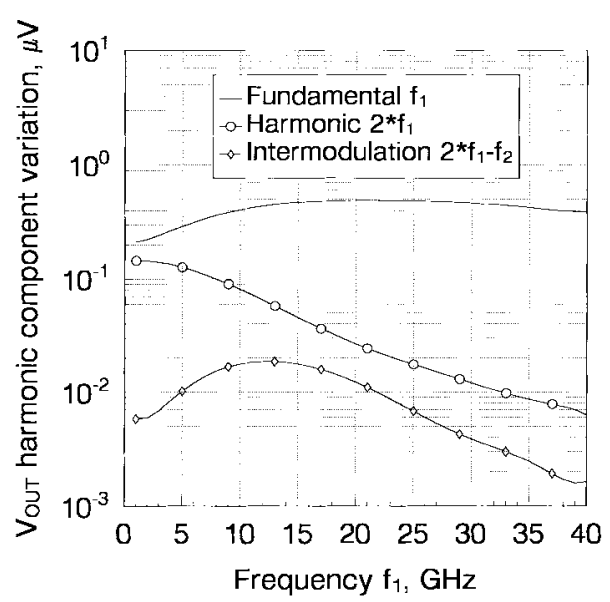

(b)

Fig. 4. Frequency $\left(f_{1}\right)$ dependence of the magnitude of the input (a) and output (b) voltage variation for the fundamental $\left(f_{1}\right)$, second harmonic $\left(2 f_{1}\right)$ and IMP $\left(2 f_{1}-f_{2}\right)$ components due to a $1 \%$ doping variation. The working point is $V_{\mathrm{DS}}=1.5 \mathrm{~V}$ and $V_{\mathrm{GS}}=-1.4 \mathrm{~V}$, and $f_{2}=f_{1}+100 \mathrm{MHz}$; the peak amplitude of the input tones is $0.3 \mathrm{~V}$.

reducing the level of third-order IMP's with respect to the fundamental frequency on a proper range of input power levels. In practice, there are several possibilities as far as the optimization goal has to be chosen. For example, one could assume as the optimization goal the maximum device gain and minimum third-order IMP levels for medium input power, keeping in mind a small-signal amplifier with maximum linearity. Conversely, the optimization goal could be selected as the maximum output power and minimum third-order IMP's for input power levels close to the device compression point, thus implying the simultaneous optimization of the device load. In both cases, device stability should be ensured throughout optimization. As a case study, the authors considered a simpler but practically significant goal: starting from a standard 50- $\Omega$ load at the device input and output ports, the authors optimized the ratio between the output power at the fundamental frequency and the third-order IMP's.

The device considered is a two-implant low-power MESFET, with $0.5-\mu \mathrm{m}$ gate length, $100-\mu \mathrm{m}$ gate width, and $1-\mu \mathrm{m}$ 


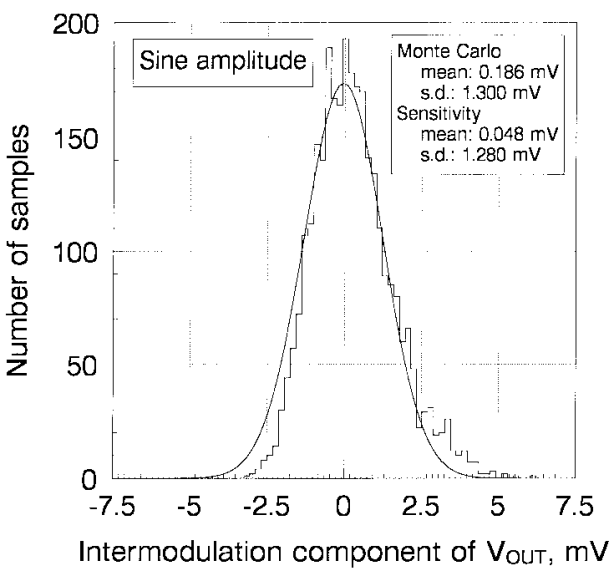

(a)

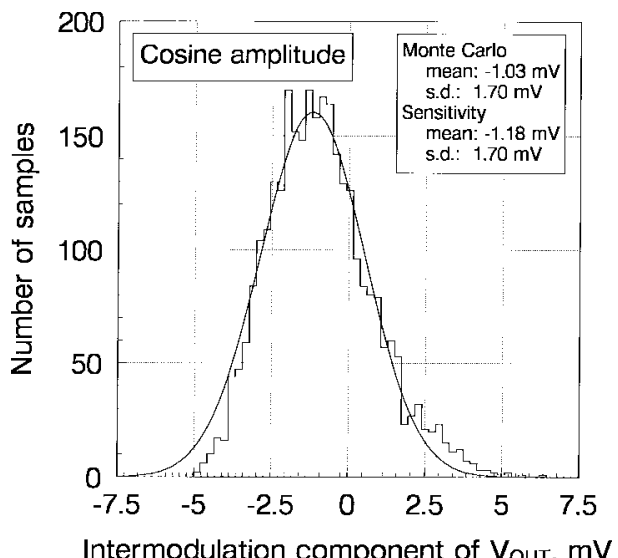

(b)

Fig. 5. Statistical analysis of the amplitude of the sine (a) and cosine (b) component of the output voltage for a $0.5-\mu \mathrm{m}$ gate length MESFET due to random variations of the implant energy with respect to its nominal value. The continuous curve is the sensitivity model, the histogram the distribution obtained through Monte Carlo analysis carried out directly on the physical model. The working point is $V_{\mathrm{DS}}=1.5 \mathrm{~V}$ and $V_{\mathrm{GS}}=-1.4 \mathrm{~V}$, the input frequencies are $f_{1}=10 \mathrm{GHz}$ and $f_{2}=f_{1}+100 \mathrm{MHz}$; the peak amplitude of the input tones is $0.5 \mathrm{~V}$.

spacing between gate and drain (source) electrodes. The device was biased at $V_{\mathrm{GS}}=-1.4 \mathrm{~V}, V_{\mathrm{DS}}=3 \mathrm{~V}$ and then excited with a two-tone input signal of frequencies $f_{1}=5 \mathrm{GHz}$, $f_{2}=f_{1}+100 \mathrm{MHz}$, and variable amplitude, so as to achieve an input power varying between -70 and $-15 \mathrm{~dB}$. The optimization of the doping profile was carried out by minimizing the ratio between the fundamental and third-order IMP power levels for several input powers chosen between $-30 \mathrm{~dB}$ and the $1 \mathrm{~dB}$ compression point. The optimization parameters were chosen as in [9] and the same optimization technique was used.

As the starting point for the optimization process, the authors chose an implanted doping profile which had already been optimized (see [9]) in order to achieve the maximum linearity in the transfer characteristics for $V_{\mathrm{DS}}=3 \mathrm{~V}$ (that is, maximally constant $g_{m}$ ). The doping profiles of the initial and optimized devices are shown in Fig. 7, together with the constant profile of an epitaxial device of the same geometry, discussed in [9] and introduced here for comparison. While

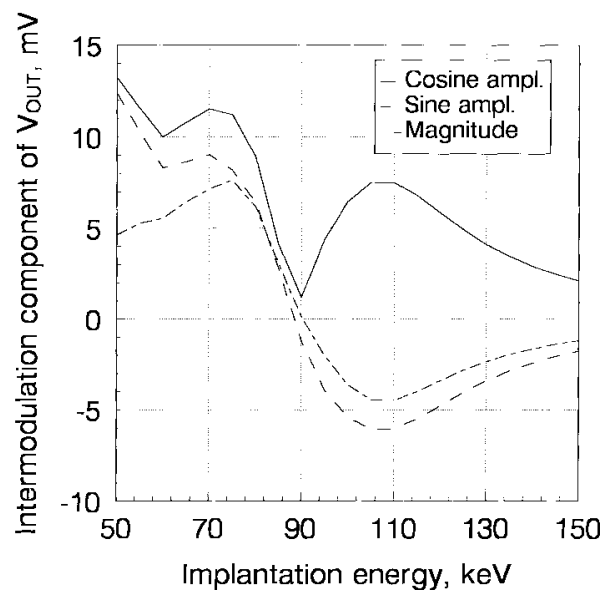

Fig. 6. Energy dependence of the amplitude of the sine and cosine components and of the magnitude of the IMP $\left(2 f_{1}-f_{2}\right)$ of the output voltage for a $0.5-\mu \mathrm{m}$ MESFET. The working point is $V_{\mathrm{DS}}=1.5 \mathrm{~V}$ and $V_{\mathrm{GS}}=-1.4 \mathrm{~V}$, the input frequencies are $f_{1}=10 \mathrm{GHz}$ and $f_{2}=f_{1}+100 \mathrm{MHz}$; the peak amplitude of the input tones is $0.5 \mathrm{~V}$.

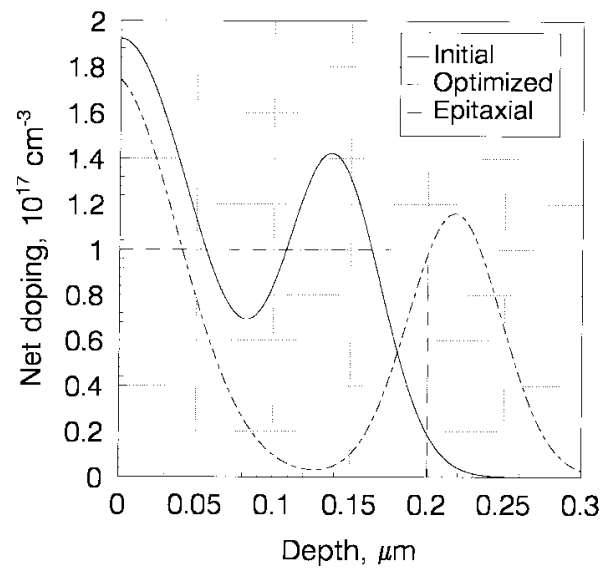

Fig. 7. Doping profile for the epitaxial device, the device optimized for maximum linearity and the device optimized to minimize the IMP.

both optimized profiles qualitatively follow the simple rule, according to which good linearity can be obtained with a profile increasing toward the substrate (ideally, a delta profile), the IMP performances of the LS optimized device are much better than the ones of the device with maximally constant $g_{m}$, despite the higher gain of the latter (about $4 \mathrm{~dB}$ ). The resulting $P_{\mathrm{IN}}-P_{\mathrm{OUT}}$ curves for the fundamental output frequency and third-order IMP are shown in Fig. 8; the small signal operating gain of the optimized device is around $14 \mathrm{~dB}$. For input power up to $-30 \mathrm{~dB}$ the IMP level of the optimized device is approximately $30 \mathrm{~dB}$ under the "maximally linear" MESFET. For comparison, the authors also show the performances of the epitaxial device, which is used in [9] as the starting point for the optimization with respect to the transconductance. Despite having an almost quadratic transfer characteristics, this device exhibits slightly better IMP performances than the $g_{m}$-optimized MESFET. The central processing unit (CPU) time for the whole optimization process was $3570 \mathrm{~s}$ on a HP735/125 workstation; throughout the optimization, the 


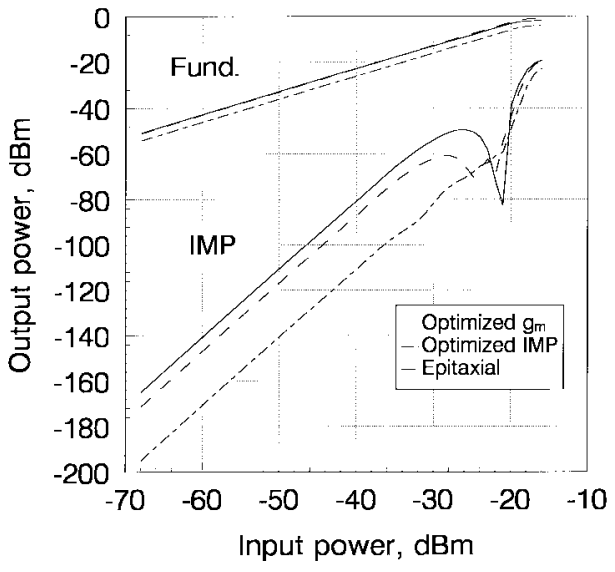

Fig. 8. $\quad P_{\mathrm{IN}}-P_{\mathrm{OUT}}$ characteristics for a two-tone excitation. The two fundamentals $\left(f_{1}=5 \mathrm{GHz}, f_{2}=5.1 \mathrm{GHz}\right)$ and the third-order IMP $\left(2 f_{1}-f_{2}\right)$ are shown for the epitaxial, the maximum dc linearity, and the IMP-optimized device. The difference between the fundamental component of $P_{\text {OUT }}$ of the epitaxial and $g_{m}$ optimized devices is small and cannot be appreciated with the chosen scale.

MESFET was discretized with a nonuniform triangular mesh of about 1500 nodes.

\section{CONCLUSION}

An efficient technique for the physics-based sensitivity analysis of microwave devices and circuits under LS quasiperiodic excitation has been presented. The proposed approach is based on the "off-line" use of circuit-oriented intermediate LS models to link circuit performances to physics-based simulations carried out with multidimensional transport models. The LS circuit-oriented sensitivity model is characterized on the basis of $\mathrm{dc}$ and small-signal ac device sensitivities computed in several working points; if a quasi-static charge-control model is used for the active device, only the sensitivities of the dc currents and charges are needed, thus enabling a very efficient look-up table based implementation. Results have been presented on examples of statistical analysis of the intermodulation performances of a MESFET under two-tone excitation with respect to random variations of the implant energy, and a doping profile optimization study has been developed to achieve minimum third-order IMP's. The proposed approach enables the efficiently carrying out of the physics-based sensitivity analysis of circuits with one or more active devices, while preserving the accuracy and flexibility offered by physics-based multidimensional device models.

\section{REFERENCES}

[1] IEEE Trans. Microwave Theory Tech., Special Issue on "Processoriented microwave CAD modeling," vol. 40, July 1992.

[2] F. Filicori, G. Ghione, and C. Naldi, "Physics-based electron device modeling and computer-aided MMIC design," IEEE Trans. Microwave Theory Tech., vol. 40, pp. 1333-1352, July 1992.

[3] J. W. Bandler, R. M. Biernacki, Q. Cai, S. H. Chen, S. Ye, and Q.J. Zhang, "Integrated physics-oriented statistical modeling, simulation and optimization," IEEE Trans. Microwave Theory Tech., vol. 40, pp. 1374-1400, July 1992.
[4] A. Gnudi, P. Ciampolini, R. Guerrieri, M. Rudan, and G. Baccarani, "Sensitivity analysis for device design," in Proc. ESSDERC 87, Bologna, Italy, Sept. 1987, pp. 551-554; also IEEE Trans. Computer-Aided Design, vol. CAD-6, pp. 879-885, Sept. 1987.

[5] S. W. Director and D. A. Rohrer, "Automated network design-The frequency-domain case," IEEE Trans. Circuit Theory, vol. 16, pp. 330-337, Aug. 1969.

[6] G. Ghione and F. Filicori, "A computationally efficient unified approach to the numerical analysis of the sensitivity and noise of semiconductor devices," IEEE Trans. Computer-Aided Design, vol. 12, p. 425-438, 1993.

[7] F. H. Branin, "Network sensitivity and noise analysis simplified," IEEE Trans. Circuit Theory, vol. CT-20, pp. 285-288, May 1973.

[8] F. Bonani, G. Ghione, M. R. Pinto, and R. K. Smith, "A novel implementation of noise analysis in general-purpose PDE-based semiconductor device simulators," in IEDM 1995, Washington, DC, Dec. 10-13, 1995, pp. 777-780.

[9] S. Donati, F. Bonani, M. Pirola, and G. Ghione, "Sensitivity-based optimization and statistical analysis of microwave semiconductor devices through multidimensional physical simulation" Int. J. Microwave and Millimeter-Wave Comput.-Aided Eng., vol. 7, no. 1, pp. 129-143, Jan. 1997.

[10] C. M. Snowden, "Numerical simulation of microwave GaAs MESFET's," in Proc. Int. Conf. Simulation Semiconductor Devices Process. Swansea, U.K.: Pineridge Press, July 1984, pp. 406-425.

[11] K. M. Whight, P. A. Gough, and P. Walker, "Large-signal periodic timedomain simulation," Int. J. Numerical Modeling: Elec. Networks, Dev. Fields, vol. 5, pp. 11-21, 1992.

[12] B. Troyanovsky, Z. Yu, and R. W. Dutton, "Large signal frequency domain device analysis via the Harmonic Balance technique," in Proc. SISDEP, Erlangen, Germany, vol. 6, pp. 114-117, 1995.

[13] F. Filicori, G. Vannini, and V. Monaco, "A nonlinear integral model of electron devices for HB circuit analysis," IEEE Trans. Microwave Theory Tech., vol. 40, pp. 1456-1465, July 1992.

[14] G. Ghione, C. Naldi, and F. Filicori, "Physical modeling of GaAs MESFET's in an integrated CAD environment: From device technology to microwave circuit performance," IEEE Trans. Microwave Theory Tech., vol. 37, pp. 457-468, Mar. 1989.

[15] S. Selberherr, Analysis and Simulation of Semiconductor Devices. Berlin, Germany: Springer-Verlag, 1984.

[16] D. E. Root, S. Fan, and J. Meyer, "Technology-independent largesignal nonquasistatic models by direct construction from automatically characterized device data," in Proc. 21st European Microwave Conf., Stuttgart, Germany, Sept. 1991, pp. 927-932.

[17] K. S. Kundert, J. K. White, and A. Sangiovanni-Vincentelli, Steady-State Methods for Simulating Analog and Microwave Circuits. Norwell, MA: Kluwer, 1990.

[18] R. Anholt, P. Balasingam, S. Y. Chou, T. W. Sigmon, and M. Deal, "Ion implantation into gallium arsenide," J. Appl. Phys., vol. 64, no. 7, pp. 3429-3438, 1988.

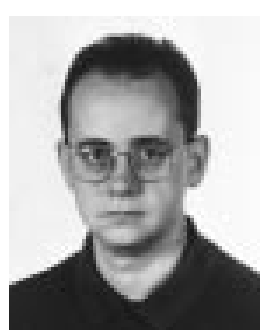

Fabrizio Bonani (S'91-M'96) was born in Torino, Italy, in 1967. He received the Laurea (cum laude), and the Ph.D. degrees in electronic engineering from Politecnico di Torino, Torino, Italy, in 1992 and 1996, respectively.

From October 1994 to June 1995, he had been with the ULSI Technology Research Department, Bell Laboratories, Murray Hill, NJ, as a Consultant, working on physics-based noise modeling of electron devices. Since 1995, he has held a researcher position in the Electronics Department, Politecnico di Torino, Italy. His research interests are mainly devoted to the physicsbased simulation of semicondutor devices, with special emphasis on the noise analysis of microwave field-effect and bipolar transistors, and to the thermal analysis of power microwave circuits. Part of his research concerns the analysis and simulation of nonlinear dynamical systems.

Dr. Bonani is member of AEI (Associazione Elettrotecnica Italiana). 


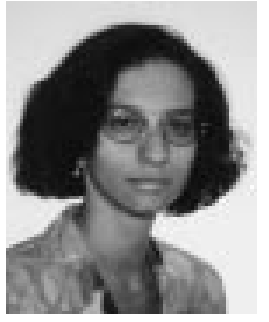

Simona Donati Guerrieri was born in Milano, Italy, on February 15, 1969. She received the Laurea degree in theoretical physics from the University of Milano, Milan, Italy, in 1993. She is presently a Ph.D. student in the Electronics Department, Politecnico di Torino, Torino, Italy, where she joined the Microwave Electronics Group

Her research interests are mainly oriented to the modeling and simulation of microwave solid state devices, including physics-based noise analysis.

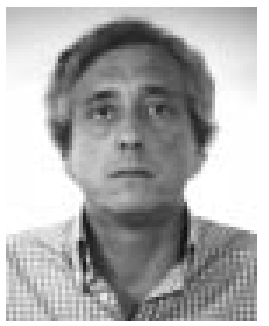

Fabio Filicori was born in Imola, Italy, in 1949 In 1974, he received the Dr. Ing. degree in electronic engineering from the University of Bologna, Bologna, Italy.

In the same year, he joined the Department of Electronics, Computer, and System Science, University of Bologna, first as an Assistant Researcher and later, as an Associate Professor of applied electronics. In 1990, he became full Professor of applied electronics on the faculty of engineering, University of Perugia, Perugia, Italy. In 1991, he joined the faculty of engineering, University of Ferrara, Ferrara, Italy, where he was full Professor responsible for the degree course in electronic engineering until November 1994. Currently, he is full Professor of industrial electronics at the University of Bologna, where he is also responsible for the Ph.D. course in electronic and computer science engineering. During his academic career, he has taught courses on computer-aided circuit design, electron devices and circuits, and industrial electronics. He has also been working as an Evaluator of applied industrial research programs for the Italian Ministry of Industry. His main research activities are in the areas of computer-aided design techniques for nonlinear microwave circuits, electron device modeling, electronic instrumentation, and industrial electronics. He was Associate Editor for a special issue on microwave CAD techniques for the journal ALTA FREQUENZA.

Dr. Filicori received the Research Award from AEI (Associazione Elettrotecnica Italiana) in 1986.

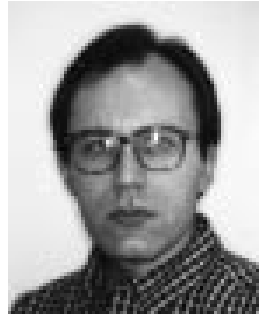

Giovanni Ghione (M'87-SM'94) was born in Alessandria, Italy, in 1956 . He received the Laurea degree (cum laude) in electronic engineering from Politecnico di Torino, Torino, Italy in 1981.

Since 1983, he had been a Research Assistant with Politecnico di Torino. From 1987 to 1990 he had been an Associate Professor at Politecnico di Milano, Milan, Italy. In 1990, he joined the University of Catania, Catania, Italy, as full Professor of electronics, and since 1991, he has been on the Faculty of Engineering as a full Professor at Politecnico di Torino, II, Torino, Italy. Since 1981, he has been engaged in Italian and European research projects (ESPRIT 255, COSMIC, and MANPOWER) in the field of active and passive microwave CAD's. His present research interests concern the physics-based simulation of active microwave and optoelectronic devices, with particular attention to noise modeling, thermal modeling, and active device optimization. His research interests also include several topics in computational electromagnetics, including coplanar component analysis. He has published more than 100 papers and book chapters in the above fields.

Mr. Ghione is a member of the editorial board of the IEEE TRANSACTIONS on Microwave Theory AND TeChNiques and member of the Associazione Elettrotecnica Italiana (AEI).

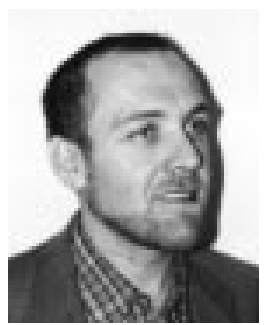

Marco Pirola was born in Velezzo Lomellina (PV), Italy, in 1963. He receiveded the Laurea degree in electronic engineering and the Ph.D. degree from Politecnico di Torino, Torino, Italy, in 1987 and 1992, respectively.

In 1992 and 1994, he was a Visiting Researcher at the Hewlett Packard Microwave Technology Division, Santa Rosa, CA. Since 1992, he has been a Researcher in the Electronics Department of Politecnico di Torino where his research concerns the simulation and modeling of microwave devices and systems. 